\title{
A MIXED QUANTUM-CLASSICAL CENTRAL LIMIT THEOREM
}

\author{
U. FRANZ \\ Institut Elie Cartan and LORIA, Université Henri Poincaré-Nancy 1 \\ B.P. 239, F-54506 Vandœuvre-lès-Nancy, France \\ and \\ Arnold Sommerfeld Institut für Math. Physik, Technische Universität Clausthal \\ Leibnizstr. 10, D-38678 Clausthal-Zellerfeld, Germany
}

\begin{abstract}
A randomized $q$-central or $q$-commutative limit theorem on a family of bialgebras with one complex parameter is shown.

1. Introduction. In [Spe92] Speicher proved a non-commutative limit theorem, in which the commutation relations of the quantum random variables are given by classical $\{-1,1\}$-valued random variables, i.e. Bernoulli random variables. We will show a similar limit theorem, but placed in a bialgebra setting. Here it is the coproduct that depends on a sequence of i.i.d. complex-valued random variables. This also leads to $q$-commutation relations for the increments, but with less independence in the choice of the commutation factors, as we will see. We will also see that the limit distribution can be expressed as an exponential with respect to a (non-associative!) averaged convolution.

We begin by briefly recalling the results of Speicher [Spe92] and Schürmann [Sch93].

In Section 2 we introduce the bialgebras used to formulate the main theorem (Section 3 ), and the algebra structure of their duals.

Finally, in Section 4, we show how explicit formulas for the moments and their associated measures can be obtained using dual representations.

Let $q \in \mathbb{C}$ be a fixed complex number, and $\tilde{x}_{1}, \tilde{x}_{2}, \ldots$ a sequence of quantum random variables that satisfy

$$
\tilde{x}_{n} \tilde{x}_{m}=q \tilde{x}_{m} \tilde{x}_{n}, \quad \tilde{x}_{n} \tilde{x}_{m}^{*}=q \tilde{x}_{m}^{*} \tilde{x}_{n}
$$

for $n>m$, and set

1991 Mathematics Subject Classification: 60F05, 16W30.

Current address: IRMA, Université Louis Pasteur, 7, Rue René Descartes, 67084 Strasbourg Cedex, France.

The paper is in final form and no version of it will be published elsewhere.
\end{abstract}




$$
\tilde{S}_{N}=\frac{\tilde{x}_{1}+\cdots+\tilde{x}_{N}}{\sqrt{N}} .
$$

If, for $n \in \mathbb{N}$, we have e.g. $\left\langle\Omega, \tilde{x}_{n} \Omega>=<\Omega, \tilde{x}_{n}^{*} \Omega>=<\Omega, \tilde{x}_{n}^{2} \Omega>=<\Omega,\left(\tilde{x}_{n}^{*}\right)^{2} \Omega>=\right.$ $<\Omega, \tilde{x}_{n} \tilde{x}_{n}^{*} \Omega>=0,<\Omega, \tilde{x}_{n}^{*} \tilde{x}_{n} \Omega>=g \in \mathbb{R}_{+}$in the vacuum state $\Omega$, then the moments of $\left(S_{N}, S_{N}^{*}\right)$ (in the state $\Omega$ ) converge for $N \rightarrow \infty$ to those of the quantum Azéma process, cf. [Sch91].

If we take instead quantum random variables $\hat{x}_{1}, \hat{x}_{2}, \ldots$, whose commutation relations are determined by classical i.i.d. random variables $\left(\varepsilon_{m n}\right)_{n, m \in \mathbb{N}}, P\left(\varepsilon_{n m}=\varepsilon_{m n}=+1\right)=p$, $P\left(\varepsilon_{n m}=\varepsilon_{m n}=-1\right)=1-p, p \in[0,1]$,

$$
\hat{x}_{n} \hat{x}_{m}=\varepsilon_{n m} \hat{x}_{m} \hat{x}_{n}
$$

(no relations between $\hat{x}_{n}$ and $\left.\hat{x}_{m}^{*}\right)$ and set again $\hat{S}_{N}=\left(\hat{x}_{1}+\cdots+\hat{x}_{N}\right) / \sqrt{N}$, then the moments of $\left(\hat{S}_{N}, \hat{S}_{N}^{*}\right)$ converge for $N \rightarrow \infty$ to those of Bożejko and Speicher's $q$-Brownian motion [BS91, BKS96], see [Spe92]. The parameters $q$ and $p$ are related via $q=2 p-1$.

Define

$$
\omega=\left(\begin{array}{l}
0 \\
1
\end{array}\right), \quad x=\left(\begin{array}{ll}
0 & 1 \\
0 & 0
\end{array}\right), \quad y(q)=\left(\begin{array}{ll}
1 & 0 \\
0 & q
\end{array}\right),
$$

for $q \in \mathbb{C}$. Then we can realize the quantum random variables $\left(\tilde{x}_{n}\right)_{n \in \mathbb{N}}$ and $\left(\hat{x}_{n}\right)_{n \in \mathbb{N}}$ on the infinite tensor product $\left(\mathbb{C}^{2}\right)^{\otimes \mathbb{N}, \omega}$ of $\mathbb{C}^{2}$ (as a Hilbert space, the product being taken with respect to the sequence $\left.\omega_{n}=\omega\right)$ as

$$
\begin{aligned}
& \tilde{x}_{n}=\underbrace{y(q) \otimes \cdots \otimes y(q)}_{n-1 \text { times }} \otimes x \otimes 1 \otimes 1 \otimes \cdots, \\
& \hat{x}_{n}=y\left(\varepsilon_{n, 1}\right) \otimes \cdots \otimes y\left(\varepsilon_{n, n-1}\right) \otimes x \otimes 1 \otimes 1 \otimes \cdots .
\end{aligned}
$$

The state is given by $\Omega=\omega^{\otimes \mathbb{N}}$.

In this realization the following amounts to replacing the parameter $q$ in the definition of $\tilde{x}_{n}$ by complex-valued random variables $\left(q_{i}\right)_{i \in \mathbb{N}}$, i.e. we set $x_{n}=y\left(q_{1}\right) \otimes \cdots \otimes y\left(q_{n-1}\right) \otimes$ $x \otimes 1 \otimes \cdots$ But, in order to stay in a bialgebra framework, we get less independence than in the commutation factors of the $\hat{x}_{n}$. It turns out that an increment $x_{n}$ has to satisfy the same commutation relation either with all preceeding or with all following increments, i.e. we have two possible cases

$$
\begin{array}{ll}
\text { a) } \quad x_{n} x_{m}=q_{n} x_{m} x_{n} & \text { for } n>m, \\
\text { b) } \quad x_{n} x_{m}=q_{m} x_{m} n_{n} & \text { for } n>m .
\end{array}
$$

2. The family of bialgebras. Let $\mathcal{A}$ be the (unital, associative) *-algebra generated by $x, x^{*}$, and $\left\{y_{\alpha} ; \alpha \in \mathbb{C} \backslash\{0\}\right\}$ with the relations $y_{\alpha}^{*}=y_{\bar{\alpha}}$, and

$$
y_{\alpha} x=\alpha x y_{\alpha}, \quad x^{*} y_{\alpha}=\alpha y_{\alpha} x^{*}, \quad y_{\alpha} y_{\beta}=y_{\alpha \beta} \text { for } \alpha, \beta \in \mathbb{C} \backslash\{0\}, \quad y_{1}=1 .
$$

Note that $\operatorname{deg} x=\operatorname{deg} x^{*}=1, \operatorname{deg} y_{\alpha}=0$ for all $\alpha \in \mathbb{C} \backslash\{0\}$ defines a grading, and so we can introduce a scaling map $s(r): \mathcal{A} \rightarrow \mathcal{A}$, for $r \in \mathbb{R}$, by setting $s(r) a=r^{-\operatorname{deg} a} a$ on homogeneous elements.

A basis of $\mathcal{A}$ is given by

$$
\mathcal{B}=\left\{y_{\alpha} w ; w \text { a word in the two letters } x, x^{*} \text {, and } \alpha \in \mathbb{C} \backslash\{0\}\right\} .
$$


On this algebra we can define a whole family of coalgebras, depending on one parameter $q \in \mathbb{C} \backslash\{0\}$, namely,

$\Delta_{q} x=x \otimes y_{q}+1 \otimes x, \quad \Delta_{q} x^{*}=x \otimes y_{\bar{q}}+1 \otimes x^{*}, \quad \Delta_{q}\left(y_{\alpha}\right)=y_{\alpha} \otimes y_{\alpha}$ for $\alpha \in \mathbb{C} \backslash\{0\}$, and $\varepsilon_{q}(x)=\varepsilon_{q}\left(x^{*}\right)=0, \varepsilon_{q}\left(y_{\alpha}\right)=1$ for all $\alpha \in \mathbb{C} \backslash\{0\}$. In fact, this is a Hopf algebra with the antipode $S_{q}: \mathcal{A} \rightarrow \mathcal{A}$,

$$
S_{q}\left(y_{\alpha}\right)=y_{1 / \alpha}, \quad S_{q}(x)=-x y_{1 / q}, \quad S_{q}\left(x^{*}\right)=-x^{*} y_{1 / \bar{q}}
$$

Using these different coalgebra structures we obtain different multiplications for functionals on $\mathcal{A}$, i.e. a one-parameter family of convolutions,

$$
\varphi *_{q} \psi=(\varphi \otimes \psi) \circ \Delta_{q}
$$

for linear functionals $\varphi, \psi: \mathcal{A} \rightarrow \mathbb{C}$.

For a word $w$ in the two letters $x, x^{*}$, let $\chi(w)$ be the functional defined by

$$
\chi(w)\left(y_{\beta} w^{\prime}\right)= \begin{cases}1 & \text { if } w=w^{\prime}, \\ 0 & \text { else }\end{cases}
$$

on the basis $\mathcal{B}$. Then $\mathcal{U}=\operatorname{span}\left\{\chi(w) ; w\right.$ a word in the two letters $\left.x, x^{*}\right\}$ is a subalgebra of the dual of $\mathcal{A}$ (with the multiplication $m_{q}=\Delta_{q}^{*}$ ). Let $a \in \mathcal{A}, \Delta_{q} a=\sum a_{i}^{(1)} \otimes a_{i}^{(2)}$. Then the product $\chi(w) *_{q} \chi\left(w^{\prime}\right)$ is defined by $\left(\chi(w) *_{q} \chi\left(w^{\prime}\right)\right)(a)=\sum \chi(w)\left(a_{i}^{(1)}\right) \chi\left(w^{\prime}\right)\left(a_{i}^{(2)}\right)$. We get by induction

$$
\begin{aligned}
\Delta_{q}\left(x^{n}\right) & =\sum_{\nu=0}^{n}\left[\begin{array}{l}
n \\
\nu
\end{array}\right]_{q} x^{\nu} \otimes x^{n-\nu} y_{q^{\nu}}, \\
\Delta_{q}\left(\left(x^{*}\right)^{n}\right) & =\sum_{\nu=0}^{n}\left[\begin{array}{l}
n \\
\nu
\end{array}\right]_{q}\left(x^{*}\right)^{\nu} \otimes y_{\bar{q}^{\nu}}\left(x^{*}\right)^{n-\nu},
\end{aligned}
$$

where $\left[\begin{array}{c}n \\ \nu\end{array}\right]=\frac{q_{n} !}{q_{\nu} ! q_{n-\nu} !}, q_{n} !=\prod_{\nu=1}^{n} q_{\nu}, q_{n}=\sum_{\nu=1}^{n} q^{\nu-1}=\frac{q^{n}-1}{q-1}$, and from this we can calculate the coproduct of any element of $\mathcal{A}$.

To calculate the product $\chi(w) *_{q} \chi\left(w^{\prime}\right)$, we have to see what elements of $\mathcal{A}$ have a term $y_{\alpha} w \otimes y_{\beta} w^{\prime}$ in their coproduct. Since the coproduct does not change the total number of $x^{\prime}$ 's and $x^{*}$ 's, but just splits a word into two, the product has to do the inverse. We get

$$
\chi(w) *_{q} \chi\left(w^{\prime}\right)=\sum_{v} c_{w, w^{\prime}}^{v}(q) \chi(v),
$$

where $v$ runs over all words that can be obtained by shuffling $w$ and $w^{\prime}$, and the $c_{w, w^{\prime}}^{v}$ are polynomials in $q$ and $q^{-1}$. To get the explicit expression, use the following procedure.

In the first term $v$ is simply the concatenation of $w$ and $w^{\prime}$, and the coefficient is equal to one. Then move the letters of $w$ to the right, without changing their order, and multiply by $q$ every time an $x$ is moved to the right past another $x$, and by $q^{-1}$ every time it is moved past an $x^{*}$. When moving an $x^{*}$ to the right take the conjugate factors, i.e. a $\bar{q}$ when it is moved past an $x$, and a $\bar{q}^{-1}$ when it is moved past an $x^{*}$. We get e.g.

$$
\begin{aligned}
\chi(x) *_{q} \chi(x) & =(1+q) \chi(x x), \\
\chi(x) *_{q} \chi\left(x^{*}\right) & =\chi\left(x x^{*}\right)+q^{-1} \chi\left(x^{*} x\right), \\
\chi\left(x^{*}\right) *_{q} \chi(x) & =\chi\left(x^{*} x\right)+\bar{q} \chi\left(x x^{*}\right),
\end{aligned}
$$




$$
\chi\left(x^{*}\right) *_{q} \chi\left(x^{*}\right)=\left(1+\bar{q}^{-1}\right) \chi\left(x^{*} x^{*}\right) .
$$

We will also need the averaged convolution (w.r.t. to a $\mathbb{C} \backslash\{0\}$-valued random variable q) defined by

$$
u_{1} \bar{*} u_{2}=\mathbb{E}\left(u_{1} *_{q} u_{2}\right),
$$

i.e. $u_{1} \bar{*} u_{2}$ is the functional defined by $u_{1} \bar{*} u_{2}(a)=\int\left(u_{1} \otimes u_{2}\right)\left(\Delta_{q}(a)\right) \mathrm{d} \mu(q)$, where $\mu$ is the law of $q$. This binary operation conserves positivity, but in general it is not associative.

3. The central limit theorem. We will now show that the functionals

$$
\text { a) } \begin{aligned}
\varphi_{\rightarrow}^{N} & =\left(\left(\varphi *_{q_{1}} \varphi\right) *_{q_{2}} \varphi\right) *_{q_{3}} \cdots *_{q_{N-1}} \varphi \circ s(\sqrt{N}) \\
& =(\varphi \otimes \cdots \otimes \varphi) \circ\left(\Delta_{q_{1}} \otimes \mathrm{id}^{\otimes N-2}\right) \circ \cdots \circ \Delta_{q_{N-1}} \circ s(\sqrt{N}) \\
\text { b) } \varphi_{\leftarrow}^{N} & =\varphi *_{q_{N-1}} \cdots *_{q_{3}}\left(\varphi *_{q_{2}}\left(\varphi *_{q_{1}} \varphi\right)\right) \circ(\sqrt{N}) \\
& =(\varphi \otimes \cdots \otimes \varphi) \circ\left(\mathrm{id}^{\otimes N-2} \otimes \Delta_{q_{1}}\right) \circ \cdots \circ \Delta_{q_{N-1}} \circ s(\sqrt{N})
\end{aligned}
$$

converge for appropriately chosen functionals $\varphi$.

In the realization from Section 1 with $\varphi(\cdot)=\langle\omega, \cdot \omega\rangle$ and for a non-commutative polynomial $P\left(x, x^{*}\right)$ we can write

$$
\varphi_{\leftarrow, \rightarrow}^{N}\left(P\left(x, x^{*}\right)\right)=<\Omega, P\left(S_{N}, S_{N}^{*}\right) \Omega>,
$$

with

and

$$
S_{N}=\frac{x_{1}^{(N)}+\cdots+x_{N}^{(N)}}{\sqrt{N}}
$$

$$
\begin{aligned}
& \text { a) } x_{n}^{(N)}=\underbrace{1 \otimes \cdots \otimes 1}_{n-1 \text { times }} \otimes x \otimes y\left(q_{n}\right) \otimes \cdots \otimes y\left(q_{N-1}\right) \\
& \text { b) } \quad x_{n}^{(N)}=\underbrace{1 \otimes \cdots \otimes 1}_{n-1 \text { times }} \otimes x \otimes \underbrace{y\left(q_{N-n}\right) \otimes \cdots \otimes y\left(q_{N-n}\right)}_{N-n \text { times }},
\end{aligned}
$$

where, as one verifies easily, the increments $x_{n}^{(N)}$ satisfy commutation relations of the form given at the end of Section 1.

We state now our result.

THEOREM 1. Let $\left(q_{n}\right)_{n \in \mathbb{N}}$ be i.i.d. random variables with values in $\mathbb{C} \backslash\{0\}$ such that $\mathbb{E}\left(\left|q_{1}^{m}\right|\right)<\infty$ for all $m \in \mathbb{Z}$, and let $\varphi: \mathcal{A} \rightarrow \mathbb{C}$ be a normed functional in $\mathcal{U}$. Suppose furthermore that $\varphi$ is centralized, i.e. $\varphi(x)=\varphi\left(x^{*}\right)=0$. Then the moments of

$$
\begin{aligned}
& \varphi_{\rightarrow}^{N}=\left(\left(\varphi *_{q_{1}} \varphi\right) *_{q_{2}} \varphi\right) *_{q_{3}} \cdots *_{q_{N-1}} \varphi \circ s(\sqrt{N}) \\
& \varphi_{\leftarrow}^{N}=\varphi *_{q_{N-1}} \cdots *_{q_{3}}\left(\varphi *_{q_{2}}\left(\varphi *_{q_{1}} \varphi\right)\right) \circ s(\sqrt{N})
\end{aligned}
$$

converge for $N \rightarrow \infty$ in probability to those of the functionals

$$
\begin{aligned}
& \varphi_{\rightarrow}^{\infty}=\exp _{\bar{*}, \rightarrow} g=\varepsilon+g+g \bar{*} g+(g \bar{*} g) \bar{*} g+\cdots, \\
& \varphi_{\leftarrow}^{\infty}=\exp _{\bar{*}, \leftarrow} g=\varepsilon+g+g \bar{*} g+g \bar{*}(g \bar{*} g)+\cdots,
\end{aligned}
$$

where $\left.g\right|_{\mathcal{A}^{(2)}}=\varphi$, and $\left.g\right|_{\mathcal{A}^{(k)}}=0$ for $k \neq 2$. 
Proof. We will only prove this for $\varphi_{\rightarrow}^{N}$, since the convergence of $\varphi_{\leftarrow}^{N}$ can be shown in the same way.

We can write $\varphi$ as $\varphi=\varepsilon+g+\tilde{\varphi}$, where $\tilde{\varphi}$ vanishes on homogeneous elements of degree less than three.

Let us first assume $\tilde{\varphi}=0$, i.e. $\varphi=\varepsilon+g, g=g_{1} \chi(x x)+g_{2} \chi\left(x^{*} x\right)+g_{3} \chi\left(x x^{*}\right)+$ $g_{4} \chi\left(x^{*} x^{*}\right)$. To simplify the notation we assume in fact $g=\chi\left(x^{*} x\right)$.

Then the functional

$$
\varphi_{\rightarrow}^{N}=\left(\cdots\left(\varphi *_{q_{1}} \varphi\right) *_{q_{2}} \cdots *_{q_{N-1}} \varphi\right) \circ S(\sqrt{N})
$$

can be written as

$$
\varphi_{\rightarrow}^{N}=\sum_{v} \frac{f_{v}^{N}\left(q_{1}, \ldots, q_{N-1}\right)}{N^{\frac{|v|}{2}}} \chi(v),
$$

where $|v|$ denotes the length of $v$. The coefficients $f_{v}^{N}\left(q_{1}, \ldots, q_{N-1}\right) / N^{|v| / 2}$ will be some rational functions of $q_{1}, \ldots, q_{N-1}$, as can be seen from the recurrence relations below. To prove the theorem for this case it is sufficient to show that $\mathbb{E}\left(f_{v}^{N}\left(q_{1}, \ldots, q_{N-1}\right) / N^{|v| / 2}\right)$ converges and that $\operatorname{Var}\left(f_{v}^{N}\left(q_{1}, \ldots, q_{N-1}\right) / N^{|v| / 2}\right)$ goes to zero for $N \rightarrow \infty$ (and fixed $v$ ). We will do this by induction over the length of $v$. The coefficient of 1 (the empty word) is constant and the coefficient of the only word with $|v|<4$ that occurs, i.e. $v=x^{*} x$, is equal to $N$, so the induction hypothesis is satisfied for $|v|<4$. Let $c_{w, w^{\prime}}^{v}(q)$ denote the coefficients of the multiplication, as in Equation (1). We have the following relations for $f_{v}^{N+1}\left(q_{1}, \ldots, q_{N}\right)$,

$$
f_{v}^{N+1}\left(q_{1}, \ldots, q_{N}\right)=f_{v}^{N}\left(q_{1}, \ldots, q_{N-1}\right)+\sum_{v^{\prime}:\left|v^{\prime}\right|=|v|-2} c_{v^{\prime}, x^{*} x}^{v}\left(q_{N}\right) f_{v^{\prime}}^{N}\left(q_{1}, \ldots, q_{N-1}\right),
$$

or

$$
f_{v}^{N}\left(q_{1}, \ldots, q_{N-1}\right)=\sum_{k=\frac{|v|}{2}}^{N-1} \sum_{v^{\prime}:\left|v^{\prime}\right|=|v|-2} c_{v^{\prime}, x^{*} x}^{v}\left(q_{k-1}\right) f_{v^{\prime}}^{k-1}\left(q_{1}, \ldots, q_{k-2}\right),
$$

and therefore

$$
\begin{aligned}
& \mathbb{E}\left(\frac{f_{v}^{N}\left(q_{1}, \ldots, q_{N-1}\right)}{N^{\frac{|v|}{2}}}\right) \\
& =\frac{1}{N} \sum_{k=\frac{|v|}{2}}^{N-1} \sum_{v^{\prime}:\left|v^{\prime}\right|=|v|-2} \mathbb{E}\left(c_{v^{\prime}, x^{*} x}^{v}\left(q_{k-1}\right)\right) \mathbb{E}\left(\frac{f_{v^{\prime}}^{k-1}\left(q_{1}, \ldots, q_{k-2}\right)}{N^{\frac{\left|v^{\prime}\right|}{2}}}\right) \\
& =\frac{1}{N} \sum_{v^{\prime}:\left|v^{\prime}\right|=|v|-2} \mathbb{E}\left(c_{v^{\prime}, x^{*} x}^{v}\left(q_{k-1}\right)\right) \sum_{k=\frac{|v|}{2}}^{N-1} \frac{(k-1)^{\frac{\left|v^{\prime}\right|}{2}}}{N^{\frac{\left|v^{\prime}\right|}{2}}} \mathbb{E}\left(\frac{f_{v^{\prime}}^{k-1}\left(q_{1}, \ldots, q_{k-2}\right)}{\left.(k-1)^{\frac{\left|v^{\prime}\right|}{2}}\right)}\right) \\
& \stackrel{N \rightarrow \infty}{\longrightarrow} \frac{2}{|v|} \sum_{v^{\prime}:\left|v^{\prime}\right|=|v|-2} \mathbb{E}\left(c_{v^{\prime}, x^{*} x}^{v}\left(q_{1}\right)\right) \lim _{N \rightarrow \infty} \mathbb{E}\left(\frac{f_{v^{\prime}}^{N}\left(q_{1}, \ldots, q_{N-1}\right)}{N^{\frac{\left|v^{\prime}\right|}{2}}}\right)
\end{aligned}
$$

The same technique works for the limit of the variance. Suppose $\operatorname{Var}\left(f_{v}^{N}\left(q_{1}, \ldots, q_{N-1}\right)\right)$ 
is bounded by $K_{v} N^{|v|-1}$, which is obviously true for $|v|=0,1,2,3$. Then, by

$$
\begin{aligned}
\operatorname{Var}\left(f_{v}^{N}\left(q_{1}, \ldots, q_{N-1}\right)\right) & =\operatorname{Var}\left(\sum_{k=\frac{|v|}{2}}^{N-1} \sum_{v^{\prime}:\left|v^{\prime}\right|=|v|-2} c_{v^{\prime}, x^{*} x}^{v}\left(q_{k-1}\right) f_{v^{\prime}}^{k-1}\left(q_{1}, \ldots, q_{k-2}\right)\right) \\
& \leq N \sum_{k=\frac{|v|}{2}}^{N-1} \sum_{v^{\prime}:\left|v^{\prime}\right|=|v|-2} \mathbb{E}\left(\left(c_{v^{\prime}, x^{*} x}^{v}\left(q_{k-1}\right)\right)^{2}\right) \operatorname{Var}\left(f_{v^{\prime}}^{k-1}\left(q_{1}, \ldots, q_{k-2}\right)\right) \\
& \leq N^{|v|-1} \sum_{v^{\prime}:\left|v^{\prime}\right|=|v|-2} \mathbb{E}\left(\left(c_{v^{\prime}, x^{*} x}^{v}\left(q_{1}\right)\right)^{2}\right) K_{v^{\prime}}
\end{aligned}
$$

it is true for all $v$, and therefore $\operatorname{Var}\left(f_{v}^{N}\left(q_{1}, \ldots, q_{N-1}\right) / N^{|v| / 2}\right) \stackrel{N \rightarrow \infty}{\longrightarrow} 0$. So, for $N \rightarrow \infty$, the coefficients $f_{v}^{N}\left(q_{1}, \ldots, q_{N-1}\right) / N^{v / 2}$ converge to the expectation in probability, and this limit can be calculated recursively over the order of $v$ with Equation (2). This shows that for $\varphi=\varepsilon+g$ the functionals $\varphi_{\rightarrow}^{N}$ tend to

$$
\varepsilon+g+g \bar{*} g+(g \bar{*} g) \bar{*} g+\cdots=\exp _{\bar{*}, \rightarrow}(g) .
$$

As in [Sch93, Theorem 6.1.3] the contributions of the higher order terms contained in $\tilde{\varphi}$ vanish in the limit, because they pick up higher orders of the scaling factor $N^{-1}$.

4. Special cases. We will now show how dual representations can be used to give explicit expressions for the moments of the limit functional. If $\varphi$ is a functional on a bialgebra $\mathcal{A}$, then we can associate operators $\rho_{R}(\varphi), \rho_{L}(\varphi): \mathcal{A} \rightarrow \mathcal{A}$ to it by

$$
\rho_{R}(\varphi) a=\sum a_{i}^{(1)} \varphi\left(a_{i}^{(2)}\right), \quad \rho_{L}(\varphi) a=\sum \varphi\left(a_{i}^{(1)}\right) a_{i}^{(2)}
$$

where $\Delta(a)=\sum a_{i}^{(1)} \otimes a_{i}^{(2)}$, (Sweedler's notation). The functional $\varphi$ can be retrieved from the operators since $\varphi=\varepsilon \circ \rho_{R}(\varphi)=\varepsilon \circ \rho_{L}(\varphi)$. Also, the map $\rho_{R}: \mathcal{A}^{*} \rightarrow \operatorname{Hom}(\mathcal{A})$ (resp. $\rho_{L}: \mathcal{A}^{*} \rightarrow \operatorname{Hom}(\mathcal{A})$ ) is a homomorphism (resp. anti-homomorphism), i.e.

$$
\rho_{R}(\varphi * \psi)=\rho_{R}(\varphi) \circ \rho_{R}(\psi) \quad\left(\text { resp. } \rho_{L}(\varphi * \psi)=\rho_{L}(\psi) \circ \rho_{L}(\varphi)\right) .
$$

Let $\mathcal{A}$ be as before, $\rho_{q}$ the right dual representation w.r.t. $\Delta_{q}$, and define

$$
R_{q}=\rho_{q}\left(\chi\left(x^{*} x\right)\right)=\left(\mathrm{id} \otimes \chi\left(x^{*} x\right)\right) \circ \Delta_{q}, \quad \bar{R}=\mathbb{E}\left(R_{q}\right) .
$$

These can be used to calculate the moments of $\exp _{\bar{*}, \rightarrow}$, since

$$
\varepsilon \circ \rho_{q_{1}}\left(\varphi_{1}\right) \circ \rho_{q_{2}}\left(\varphi_{2}\right) \circ \cdots \circ \rho_{q_{n}}\left(\varphi_{n}\right)=\left(\varphi_{1} *_{q_{2}} \varphi_{2}\right) *_{q_{3}} \cdots *_{q_{n}} \varphi_{n},
$$

If $q$ is real, then, for polynomials $f(z)$ in the variable $z=x+x^{*}$, we have

$$
R_{q} f(z)=\frac{f(q z)-f(z)-z(q-1) f^{\prime}(z)}{z^{2}(q-1)^{2}},
$$

i.e. $R_{q}$ is the generator of the Azéma martingale. Consult [Fra97a, Fra97b] to see how this can be derived using Hopf algebra duality and a generalized Leibniz formula. On monomials we get $R_{q} z^{n}=k_{n}(q) z^{n-2}, k_{n}(q)=\sum_{\nu=1}^{n-1} \nu q^{n-\nu}$, for $n \geq 2, R_{q} z=R_{q} 1=0$. $\bar{R}$ follows by averaging over $q$.

Thus we have the following result. 
Proposition 2. Let $q$ be a random variable with values in $\mathbb{R} \backslash\{0\}$ s.t. $\mathbb{E}\left(|q|^{n}\right)<\infty$ for all $n \in \mathbb{N}$ and denote by $\bar{*}$ the averaged convolution with respect to $q$. Then

$$
\exp _{\bar{*}, \rightarrow} \chi\left(x^{*} x\right)\left(\left(x+x^{*}\right)^{n}\right)= \begin{cases}\frac{\bar{k}_{2 m} \bar{k}_{2 m-2} \cdots \bar{k}_{2}}{m !} & \text { if } n \text { is even, } n=2 m, \\ 0 & \text { if } n \text { is odd, }\end{cases}
$$

where $\bar{k}_{n}=\sum_{\nu=1}^{n-1} \nu \mathbb{E}\left(q^{n-\nu}\right)$.

Pr o of. Apply $\exp _{\bar{*}, \rightarrow} \chi\left(x^{*} x\right)=\varepsilon \circ e^{\bar{R}}$ to $\left(x+x^{*}\right)^{n}$, which gives the desired expression since $\bar{R}\left(x+x^{*}\right)^{n}=\bar{k}_{n}\left(x+x^{*}\right)^{n-2}$.

R e m ark. Similarly, the left dual representation can be used to compute $\exp _{\bar{*}, \leftarrow}(g)$.

EXAMPLES.

- In the deterministic case, i.e. if $P\left(q=q_{0}\right)=1$ for some $q_{0} \in \mathbb{R} \backslash\{0\}$, we get the marginal distribution of the Azéma martingale with parameter $q_{0}$.

- For Bernoulli random variables, i.e. if $P(q=1)=p, P(q=-1)=1-p$, for some $p \in[0,1]$, we obtain

$$
\varphi_{\infty}\left(\left(x+x^{*}\right)^{2 m}\right)=\prod_{\mu=0}^{m-1}(1+2 \mu p)
$$

i.e. the moments of $\mu=c_{p}|x|^{\frac{1}{p}-1} e^{-x^{2} / 2 p} \mathrm{~d} x$.

\section{References}

[BKS96] M. Bożejko, B. Kümmerer and R. Speicher, q-Gaussian processes: Noncommutative and classical aspects, Commun. Math. Phys. 185 (1997), 129-154.

[BS91] M. Bożejko and R. Speicher, An example of a generalized Brownian motion, Commun. Math. Phys. 137 (1991), 519-531.

[Fra97a] U. Franz, Contribution à l'étude des processus stochastiques sur les groupes quantiques, Ph.D. Thesis, Université H. Poincaré-Nancy 1, 1997.

[Fra97b] U. Franz, Classical versions of quantum Lévy processes, in preparation.

[Sch91] M. Schürmann, Quantum q-white noise and a q-central limit theorem, Commun. Math. Phys. 140 (1991), 589-615.

[Sch93] M. Schürmann, White Noise on Bialgebras, Berlin, Springer-Verlag, 1993, Lecture Notes in Mathematics, volume 1544.

[Spe92] R. Speicher, A non-commutative central limit theorem, Math. Z. 209 (1992), 55-66. 